\title{
MMP11 promotes the proliferation and progression of breast cancer through stabilizing Smad2 protein
}

\author{
YING ZHUANG, XIANG LI, PENG ZHAN, GUOLIANG PI and GU WEN \\ Department of Breast Surgery, Hubei Cancer Hospital, Tongji Medical College, \\ Huazhong University of Science and Technology, Wuhan, Hubei 430000, P.R. China
}

Received August 30, 2020; Accepted December 8, 2020

DOI: $10.3892 /$ or.2021.7967

\begin{abstract}
Breast cancer (BC) is one of the most common malignant tumours in women. The matrix metalloproteinase (MMP) enzyme family plays a complex role in the development of BC. There is increasing evidence that MMP11 plays a major role in BC; however, the underlying mechanisms are not clear. The present study confirmed by analysing clinical samples and TCGA data sets, that high expression of MMP11 in clinical samples of $\mathrm{BC}$ was strongly associated with a poor prognosis in $\mathrm{BC}$ patients. In addition, MTT and colony formation assays indicated that the proliferative capacity of BC was affected when MMP11 expression changed. Furthermore, pathway enrichment analysis was performed and it was revealed that the TGF- $\beta$ signalling pathway was a potential downstream target of MMP11. In the TGF- $\beta$ signalling pathway, MMP11 could significantly regulate the protein expression levels of Smad 2 and Smad3 and inhibit the degradation of Smad2 through the ubiquitin proteasome pathway as determined by western blotting. In vivo, it was further verified that MMP11 knockdown could inhibit tumour proliferation and growth. Collectively, the present results demonstrated that MMP11 inhibited the degradation of Smad 2 in the TGF- $\beta$ signalling pathway, thereby promoting the development of $\mathrm{BC}$. Thus, MMP11 expression was not only revealed to be an important indicator of $\mathrm{BC}$ prognosis but may also be an important therapeutic target for further prevention of BC growth and proliferation. The present study indicated that MMP11-targeted therapy may provide new solutions for $\mathrm{BC}$ treatment.
\end{abstract}

\section{Introduction}

Breast cancer (BC) is the leading cause of cancer-related deaths among women worldwide (1). The incidence and mortality

Correspondence to: Dr Gu Wen, Department of Breast Surgery, Hubei Cancer Hospital, Tongji Medical College, Huazhong University of Science and Technology, 116 Zhuodaoquan South Road, Hongshan, Wuhan, Hubei 430000, P.R. China

E-mail: cc3e2zy@163.com

Key words: matrix metalloproteinase 11, Smad family member 2, breast cancer, proliferation, progression rates of $\mathrm{BC}$ also rank first among female malignant tumours, posing a serious threat to the health and lives of women (2). Although great progress has been made in the treatment of $\mathrm{BC}$ in recent years, the overall survival rate of $\mathrm{BC}$ patients has not improved significantly due to recurrence and metastasis, and the incidence of $\mathrm{BC}$ in China has been on the rise in the past decade (3). Therefore, increasing attention has been given to exploring relevant potential molecular markers to further improve the understanding of the pattern of BC proliferation and to develop future treatment measures to control $\mathrm{BC}$ progression.

As an important member of the matrix metalloproteinase (MMP) family, MMP11 regulates a series of physiological processes and signalling events, manipulates some biologically active molecules on the cell surface, and alters the biological behaviour of cells, playing an important role in the tumour microenvironment (4,5). Moreover, accumulated studies have revealed that MMPs are closely related to tumorigenesis and that the most functionally significant MMP is MMP11, which is overexpressed in tumours and is involved in the proliferation and malignant development of tumour cells $(6,7)$. A previous study revealed that high expression of MMP11 in BC was associated with a poor prognosis in patients (8). MMP11 was revealed to promote tumour malignancy by inhibiting apoptosis and by promoting migration and invasion, but the underlying mechanism remains unclear $(9,10)$.

In the present study, the expression level of MMP11 was first analysed in breast cancer tissues and adjacent tissues using clinical samples, and the relationship between MMP11 and clinical prognosis was explored. Furthermore, MTT and colony formation assays were used to confirm the role of MMP11 on breast cancer cell proliferation. Functional enrichment analysis and immunoprecipitation were used to confirm the mechanisms of MMP11 in the regulation of breast cancer cell proliferation. Finally, xenograft assays were further performed to demonstrate the role of MMP11 in breast cancer proliferation and growth in vivo.

\section{Materials and methods}

Patients and clinical samples. In total, 17 pairs of $\mathrm{BC}$ and paracancer (PC) tissues were collected from patients (aged 55-65 years old) at Hubei Cancer Hospital (Wuhan, China) between December 2018 and December 2019. BC patients did 
not receive any chemotherapy or endocrine therapy prior to tumour removal. Immediately after surgery, all tissues were frozen in liquid nitrogen and maintained at $-80^{\circ} \mathrm{C}$ until further analysis. In addition, three of the 17 pairs of $\mathrm{BC}$ tissues and their adjacent tissues were randomly selected for mRNA transcriptome sequencing (Illumina; PE-401-3001; Shanghai GeneChem Co., Ltd.). All clinical samples were collected with written informed consent from the patients, and this protocol was approved by the Ethics Committee of the Hubei Cancer Hospital.

Cell culture. Human breast epithelial cells (MCF10A) and human BC cell lines (MCF7, MDA231, MDA468 and MX1) were purchased from BeiNa Bio. MCF10A cells were maintained in RPMI-1640 medium supplemented with 10\% FBS (both from Gibco; Thermo Fisher Scientific, Inc.) and 1\% penicillin and streptomycin (ScienCell Research Laboratories, Inc.) in a humidified incubator with $5 \% \mathrm{CO}_{2}$ at $37^{\circ} \mathrm{C}$. $\mathrm{MCF} 7$, MDA231, MDA468 and MX1 were maintained in Dulbecco's modified Eagle's medium (DMEM) (Gibco; Thermo Fisher Scientific, Inc.) supplemented with $10 \%$ FBS and $1 \%$ penicillin and streptomycin in a humidified incubator with $5 \% \mathrm{CO}_{2}$ at $37^{\circ} \mathrm{C}$. All cell lines were confirmed to be free of mycoplasma contamination. Cycloheximide (CHX), MG132 and chloroquine, which were used for cell-based assays, were purchased from MCE. SRI-011381 (TGF- $\beta$ agonist) was purchased from Selleck Chemicals.

Transfection and infection. The MMP11 overexpression (OE) plasmid and corresponding negative control (NC) plasmid were synthesized by Shanghai GeneChem Co., Ltd. Smad family member 2 small interfering (si)RNA (si-Smad2) and the corresponding NC siRNA were purchased from Guangzhou RiboBio Co., Ltd. The sequences of siRNAs are as follows: Smad2 sense, 5'-CCAGGUGGUGAAGAAUCUATT-3', and antisense, 5'-UAGAUUCUUCACCACCUGGTT-3'; NC siRNA sense, 5'-AAAAATTCAAGACUUGGAGCU-3' and antisense, 5'-UCUUGTTUUUUUAGCUCCAAG-3'. siRNAs at a final concentration of $50 \mathrm{nM}$ and plasmids at a dose of $1.6 \mu \mathrm{g}$ per well (12-well plates) were transfected into cells at $37^{\circ} \mathrm{C}$ for $48 \mathrm{~h}$ using Lipofectamine 3000 reagent (Invitrogen; Thermo Fisher Scientific, Inc.) according to the manufacturer's protocol. Subsequent assays were carried out after $72 \mathrm{~h}$ of transfection. The MMP11 lentivirus (Lv-sh) and empty control vector (Lv-NC) were constructed by Shanghai GeneChem Co., Ltd. The aforementioned recombinant lentiviruses were stably transfected into BC cell lines (MCF7 and MDA231) with an MOI of 2, and then the transfected cells were used for subsequent experiments after 3 days of puromycin selection with a final concentration of $2 \mu \mathrm{g} / \mathrm{ml}$.

3-(4,5)-Dimethylthiahiazo(-z-yl)-3,5-di-phenyltetrazolium bromide (MTT) assay for cell proliferation. To assess cell viability, the cells were plated in 96-well plates at a density of $5 \times 10^{3}$ cells/well, and then subjected to the MTT assay (Sigma-Aldrich; Merck KGaA). Then, $20 \mu \mathrm{l}$ of MTT solution $(5 \mathrm{mg} / \mathrm{ml})$ was added to each well. After $4 \mathrm{~h}$ of incubation at $37^{\circ} \mathrm{C}$, the culture medium in the well was removed, $150 \mu 1$ dimethyl sulfoxide (DMSO) was added to each well, and the optical density was measured at $490 \mathrm{~nm}$.
The experiment was replicated three times independently. Each condition was plated in triplicate.

Colony formation assay. Seventy-two hours after transfection of siRNA or MMP11 lentivirus, 500 cells were inoculated into 6 -well plates and grown in complete medium for two weeks. Colonies were fixed with $4 \%$ paraformaldehyde at room temperature for $15 \mathrm{~min}$ and stained with $0.1 \%$ crystal violet at room temperature for $30 \mathrm{~min}$ in $100 \%$ ethanol and then counted and photographed (D850; Nikon Corporation). All colony formation assays were conducted in triplicate.

Western blot analysis and immunohistochemistry. Protein lysates of tumour tissues or cultured cells were prepared using RIPA buffer (Sigma-Aldrich; Merck KGaA) containing 1\% phenylmethanesulfonyl fluoride (PMSF) and phosphatase inhibitors. Western blot analysis was performed as previously described (11). The antibodies used were as follows: MMP11 (product code ab119284; 1:1,000 dilution; Abcam), Smad2 (cat. no. 12570-1-AP; 1:1,000 dilution), Smad3 (cat. no. 25494-1-AP; 1:1,000 dilution), and $\beta$-actin (cat. no. 20536-1-AP; 1:1,000 dilution) primary antibodies and goat anti-rabbit IgG secondary antibody (cat. no. SA00001-2; 1:5,000 dilution; all from ProteinTech Group, Inc.). For immunohistochemistry (IHC), the aforementioned antibodies MMP11 and Smad2 (1:1,000 dilution) were used. The specific immunohistochemistry protocol was performed as previously described (12).

$R N A$ isolation, reverse transcription-quantitative ( $R T-q P C R$ ). Total RNA from tissues or cultured cell lines was extracted using TRIzol ${ }^{\circledR}$ reagent (Invitrogen; Thermo Fisher Scientific, Inc.) according to the manufacturer's instructions. RT-qPCR was carried out using the Prime-Script RT kit (TaKaRa Biotechnology Co., Ltd.). Gene expression levels were established by RT-qPCR using SYBR Premix Ex Taq (TaKaRa Biotechnology Co., Ltd.) and normalized to the endogenous level of $\beta$-actin as a control. The thermocycling conditions were as follows: Initial denaturation at $95^{\circ} \mathrm{C}$ for $30 \mathrm{sec}$, and then the PCR reactions: $95^{\circ} \mathrm{C}$ for $5 \mathrm{sec}, 60^{\circ} \mathrm{C}$ for $35 \mathrm{sec}$, and 60 cycles. The primers used were as follows: MMP11 forward, 5'-CCATGT AATATCTAGATAAGGTCGGA-3' and reverse, 5'-GAGTCA AGGTCGGGTGCGTGGGAAG-3'; $\beta$-actin forward 5'-CCA AGGCCAACCGCGAGAAGATGAC-3' and reverse 5'-AGG GTACATGGTGGTGCCGCCAGAC-3'. All these experiments were performed in triplicate on a Bio-Rad CFX96 instrument (Bio-Rad Laboratories, Inc.), and the relative expression values were calculated using the $2^{-\Delta \Delta \mathrm{Cq}}$ method (12).

Immunoprecipitation. MCF7 cells were transfected as previously indicated and then lysed in lysis buffer. The samples were centrifuged at $4000 \mathrm{xg}$ at $4^{\circ} \mathrm{C}$ for $10 \mathrm{~min}$, and the supernatants were retained. Then, the supernatants were incubated with anti-MMP11 $(1 \mu \mathrm{g} / \mathrm{ml})$ or IgG $(1 \mu \mathrm{g} / \mathrm{ml}$; product no. 6990 ; Cell Signaling Technology, Inc.) at $4^{\circ} \mathrm{C}$ for $4 \mathrm{~h}$ and protein A/G-Sepharose beads $(10 \mu \mathrm{l})$ for $2 \mathrm{~h}$ at $4^{\circ} \mathrm{C}$. The immunocomplexes were washed three times, boiled in sample buffer, and subjected to western blotting with anti-Smad 2 antibody.

Xenograft assay. MCF7 cells (3x10\%/mouse, 5/group) transfected with Lv-MMP11 or Lv-NC were injected subcutaneously into 


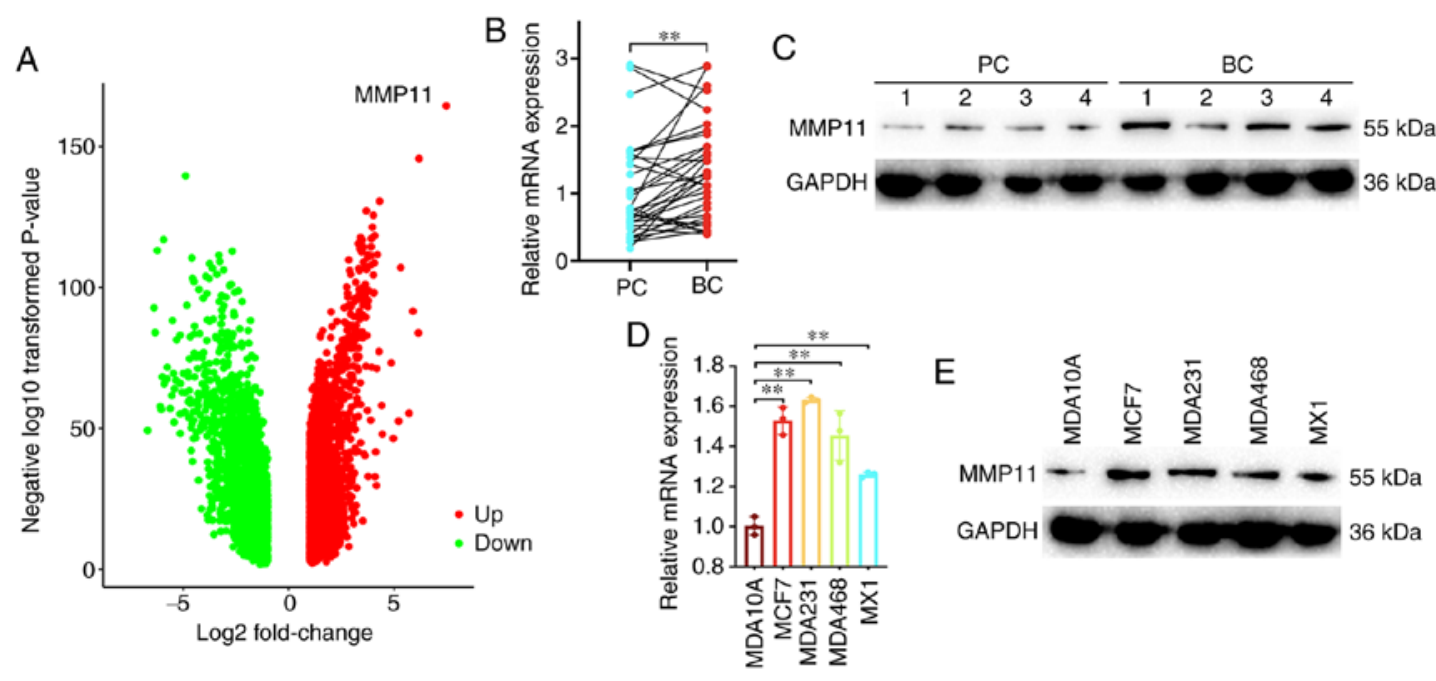

Figure 1. (A) Volcano plot of differentially expressed genes based on our transcriptome sequencing data. The expression levels of MMP11 in 34 pairs of BC and PC tissues were indicated by (B) RT-qPCR and (C) western blot assays. In addition, the expression levels of MMP11 in breast epithelial cells (MCF10A) and BC cell lines (MCF7, MDA231, MDA468 and MX1) were indicated by (D) RT-qPCR and (E) western blot assays. All data are presented as the mean \pm SD of three independent experiments. ${ }^{* *} \mathrm{P}<0.01$. MMP, matrix metalloproteinase; BC, breast cancer; PC, paracancer; RT-qPCR, reverse transcription-quantitative PCR.

the right flank region of male BALB/c nude mice (6 weeks old; weight, $\sim 15 \mathrm{~g} ; 10$ in total). All nude mice were kept in a Specific Pathogen Free (SPF) environment with controllable light (12-h light/dark cycle), temperature and humidity, with food and water available ad libitum. The mice were monitored weekly and the tumour volume was assessed; the long diameter of the tumour did not exceed $2 \mathrm{~cm}$. The formula for the tumour volume was $\mathrm{V}=\mathrm{LxW}^{2} \mathrm{x} \pi / 6$ (V, volume; L, long diameter of the tumour; $\mathrm{W}$, short diameter of the the tumour). Approximately 4 weeks after injection, according to institutional ethical guidelines, the mice were anesthetized with isoflurane and sacrificed by humanely cervical dislocation, and the weights of the tumours were recorded. The mouse experiments and the handling of animals were performed according to the Institutional and Animal Care and Use Committee of Hubei Cancer Hospital and the NIH Guide for the Care and Use of Laboratory Animals.

Bioinformatics analysis. The R 'limma' Bioconductor package was used to screen the differentially expressed genes (DEGs) between KIRC and adjacent tissues based on the following criteria: Fold change (FC), $|\log 2(\mathrm{FC})|>1$; and false discovery rate $(\mathrm{FDR})<0.05$. Adjusted $\mathrm{P}<0.05$ was used to define a gene as a DEG. An online database (https://www.UniProt.org) was used to predict MMP11-related molecules. The Database for Annotation, Visualization and Integrated Discovery (DAVID) v6.8 (david-d. ncifcrf.gov/) was used to analyze functional enrichment among DEGs. In addition, only those Kyoto Encyclopedia of Genes and Genomes (KEGG) pathways with $\mathrm{P} \leq 0.05$ and $\geq 10$ enriched genes were considered significant. Online database analysis (http://kmplot.com) was used to further investigate the relationship between MMP11 and the prognosis of $\mathrm{BC}$ patients.

Statistical analysis. All statistical analyses were performed using SPSS 22.0 (IBM Corp.), and figures were produced using GraphPad Prism 6.0 (GraphPad Software, Inc). For parametric variables, paired and unpaired Student's t-test and one-factor ANOVA followed by Tukey's post hoc test were utilized to analyse the results of the animal experiments. Survival was assessed using the Kaplan-Meier method and analysed using the log-rank test. Univariate analyses were carried out using the Cox proportional hazards model. All experimental data are expressed as the mean \pm SD of at least five independent experiments. In addition, the BC data set of The Cancer Genome Atlas (TCGA) (http://cancergenome.nih.gov/) of the University of North Carolina TCGA Genome Characterization Center was used for clinical correlation analysis (13). The differences were considered statistically significant when $\mathrm{P}<0.05$.

\section{Results}

Overexpression of MMP11 in BC tissues. To explore gene expression patterns, transcriptome sequencing of clinical $\mathrm{BC}$ samples and their adjacent tissues was performed. The sequencing results revealed that MMP11 was the most upregulated gene (Fig. 1A). To confirm the gene overexpression of MMP11, 34 paired of BC and adjacent PC tissues were used. Based on the RT-qPCR analysis, MMP11 mRNA was overexpressed in tumour tissues (Fig. 1B). Western blotting was also performed in paired $\mathrm{BC}$ and $\mathrm{PC}$ tissues, and comparison of the expression levels revealed that MMP11 was highly expressed in tumour tissues (Fig. 1C). In addition, the expression level of MMP11 was significantly higher in the four BC (MCF7, MDA231, MDA468, MX1) cell lines than in the normal human breast epithelial cell line (MDA10A) (Fig. 1D and E).

MMP11 expression is significantly associated with BC progression and poor prognosis. First, $34 \mathrm{BC}$ specimens were assessed by RT-qPCR and IHC, and it was revealed that the mRNA and protein expression levels of MMP11 were basically the same (Fig. 2A). Then, patient clinicopathological characteristics were analysed to determine the association between MMP11 expression and clinical factors. The results revealed that only tumour stage was significantly associated with MMP11 expression ( $\mathrm{P}=0.037)$ (Table I). Additionally, Kaplan-Meier survival analysis confirmed that high MMP11 
Table I. Clinical significance of MMP11 in BC.

\begin{tabular}{|c|c|c|c|c|}
\hline & MMP11 & High expression & Low expression & P-value \\
\hline \multirow{2}{*}{ Age (years) } & $>60$ & 7 & 8 & 0.73 \\
\hline & $<60$ & 10 & 9 & \\
\hline \multirow[t]{2}{*}{ Neoplasm subdivision } & Left & 9 & 10 & 0.73 \\
\hline & Right & 8 & 7 & \\
\hline \multirow[t]{2}{*}{ Oestrogen receptor } & Positive & 12 & 14 & 0.419 \\
\hline & Negative & 5 & 3 & \\
\hline \multirow[t]{2}{*}{ Progesterone receptor } & Positive & 10 & 11 & 0.724 \\
\hline & Negative & 7 & 6 & \\
\hline \multirow[t]{2}{*}{ HER2 receptor } & Positive & 9 & 5 & 0.163 \\
\hline & Negative & 8 & 12 & \\
\hline \multirow[t]{2}{*}{ Menopause status } & Pre & 3 & 7 & 0.132 \\
\hline & Post & 14 & 10 & \\
\hline \multirow[t]{2}{*}{ Pathologic stage } & $0-1$ & 7 & 13 & 0.037 \\
\hline & $2-3$ & 10 & 4 & \\
\hline \multirow[t]{2}{*}{$\mathrm{T}$} & $0-1$ & 9 & 14 & 0.067 \\
\hline & $2-3$ & 8 & 3 & \\
\hline \multirow[t]{2}{*}{$\mathrm{N}$} & $0-1$ & 12 & 12 & 1 \\
\hline & $2-3$ & 5 & 5 & \\
\hline \multirow[t]{2}{*}{ M } & 0 & 15 & 17 & 0.145 \\
\hline & 1 & 2 & 0 & \\
\hline
\end{tabular}

Bold indicates statistical significance. MMP, matrix metalloproteinase; BC, breast cancer; $\mathrm{T}$, tumour stage; $\mathrm{N}$, lymph node status; M, metastasis status.

A
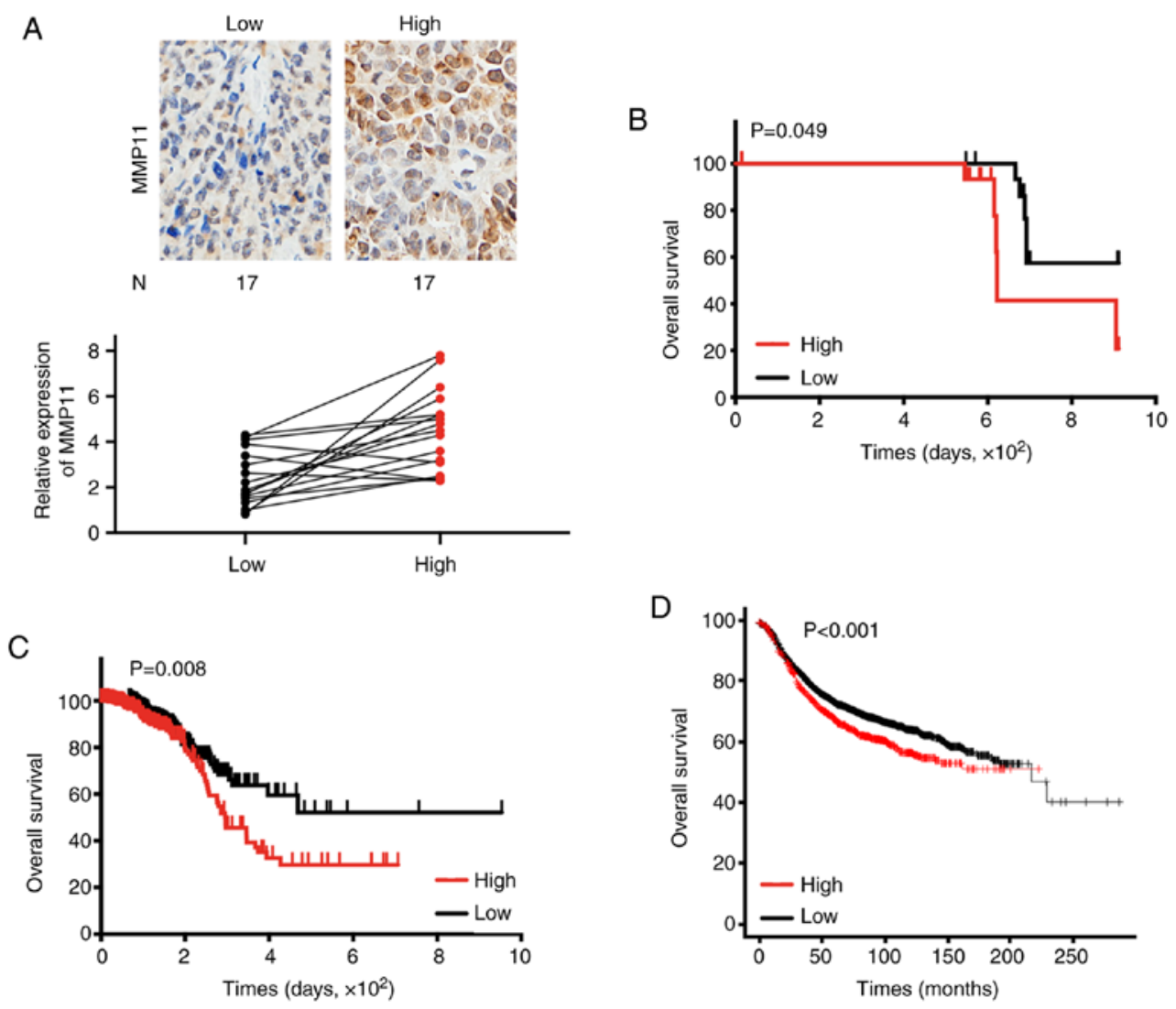

Figure 2. In total, 34 BC patients were divided into a high-MMP11 group and a low-MMP11 group according to (A) RT-qPCR and IHC data, and (B) the overall survival of each group is represented in Kaplan-Meier curves. Based on (C) the TCGA data set and (D) the online database KMplot (http://kmplot.com), $\mathrm{BC}$ patients were stratified according to the expression of MMP11 in BC tissues and Kaplan-Meier curves were generated. BC, breast cancer; MMP, matrix metalloproteinase; RT-qPCR, reverse transcription-quantitative PCR; IHC, immunohistochemistry. 
Table II. Clinical significance of MMP11 in BC based on TCGA data.

\begin{tabular}{|c|c|c|c|c|}
\hline & MMP11 & High expression & Low expression & P-value \\
\hline \multirow[t]{2}{*}{ Age (years) } & $>60$ & 186 & 201 & 0.492 \\
\hline & $<60$ & 170 & 203 & \\
\hline \multirow[t]{2}{*}{ Sex } & Female & 387 & 379 & 0.764 \\
\hline & Male & 5 & 4 & \\
\hline \multirow[t]{2}{*}{ Neoplasm subdivision } & Left & 208 & 206 & 0.839 \\
\hline & Right & 184 & 177 & \\
\hline \multirow[t]{2}{*}{ Oestrogen receptor } & Positive & 299 & 290 & 0.756 \\
\hline & Negative & 85 & 87 & \\
\hline \multirow[t]{2}{*}{ Progesterone receptor } & Positive & 267 & 235 & 0.045 \\
\hline & Negative & 117 & 140 & \\
\hline \multirow[t]{2}{*}{ HER2 receptor } & Positive & 72 & 54 & 0.027 \\
\hline & Negative & 196 & 231 & \\
\hline \multirow[t]{2}{*}{ Menopause status } & Pre & 98 & 94 & 0.480 \\
\hline & Post & 231 & 250 & \\
\hline \multirow[t]{2}{*}{ Pathologic stage } & $0-1$ & 248 & 279 & 0.004 \\
\hline & $2-3$ & 133 & 95 & \\
\hline \multirow[t]{2}{*}{$\mathrm{T}$} & $0-1$ & 279 & 315 & 0.003 \\
\hline & $2-3$ & 112 & 77 & \\
\hline \multirow[t]{2}{*}{$\mathrm{N}$} & $0-1$ & 319 & 301 & 0.320 \\
\hline & $2-3$ & 66 & 75 & \\
\hline \multirow[t]{2}{*}{ M } & 0 & 339 & 336 & 0.655 \\
\hline & 1 & 10 & 8 & \\
\hline
\end{tabular}

Bold indicates statistical significance. MMP, matrix metalloproteinase; BC, breast cancer; TCGA, The Cancer Genome Atlas; T, tumour stage; $\mathrm{N}$, lymph node status; $\mathrm{M}$, metastasis status.

expression was associated with poor OS in BC patients (Fig. 2B). To further expand the clinical samples and analyse the clinical significance of MMP11, the BC dataset from TCGA was downloaded and analysed. As revealed in Table II, a systematic analysis revealed that high MMP11 expression was significantly associated with progesterone receptor status $(\mathrm{P}=0.045)$, HER2 receptor status $(\mathrm{P}=0.027)$, tumour stage $(\mathrm{P}=0.003)$, and pathologic stage $(\mathrm{P}=0.004)$ in $\mathrm{BC}$. Moreover, Kaplan-Meier survival analysis confirmed that the OS rate of patients with low MMP11 expression was significantly higher than that of patients with high MMP11 expression $(\mathrm{P}=0.008)$ (Fig. 2C). Online database analysis (http://kmplot.com) also confirmed that high expression of MMP11 was closely related to poor OS in $\mathrm{BC}(\mathrm{P}<0.001)$ (Fig. 2D) (14).

Overexpression of MMP11 promotes the proliferation of $B C$ cells. To explore whether MMP11 affects the biological behaviour of $\mathrm{BC}$, cell proliferation and colony formation assays were applied. First, it was determined that OE plasmid transfection significantly increased the mRNA and protein expression of MMP11 in MCF7 and MDA231 cells compared with that in the control group (Fig. 3A). Further results revealed that MMP11 overexpression significantly increased the proliferation and colony formation of MCF7 and MDA231 cells (Fig. 3B and C). In addition, to further demonstrate the role of MMP11 in BC, MMP11 Lv-shRNA was used to determine whether MMP11 knockdown could inhibit BC cell development. Lv-shRNA significantly reduced the expression of MMP11 mRNA and protein (Fig. 3D). Moreover, knockdown of MMP11 markedly inhibited the proliferation and colony formation of MCF7 and MDA231 cells (Fig. 3E and F). Therefore, these results indicated that overexpression of MMP11 promoted the proliferation of BC cells.

MMP11 regulates the TGF- $\beta$ signalling pathway. To further investigate the biological importance of MMP11 in BC, we used an online database (https://www.UniProt.org) to predict MMP11-related molecules and performed Gene Ontology (GO) and Kyoto Encyclopedia of Genes and Genomes (KEGG) analyses (https://david-d.ncifcrf.gov) $(15,16)$. The biological processes of MMP11-related molecules were mainly enriched in 'cell-cell signalling', 'regulation of cell proliferation', and 'cell-cell adhesion' (Fig. 4A). In addition, certain signalling pathways associated with breast carcinogenesis were identified to be related to MMP11 in the KEGG pathway analysis; these pathways included the 'TGF- $\beta$ signalling pathway', 'PPAR signalling pathway', 'apoptosis' pathway and 'pathways in cancer' (Fig. 4B). Then, the TGF- $\beta$ signalling pathway, which was the most closely related to cell growth and proliferation in our analysis, was investigated. Smad2 and Smad3 are two key molecules in the TGF- $\beta$ signalling pathway (17). The present results revealed that MMP11 overexpression increased the expression of Smad2 and Smad3 (Fig. 4C), while knockdown of MMP11 inhibited Smad2 and Smad3 expression (Fig. 4D), 

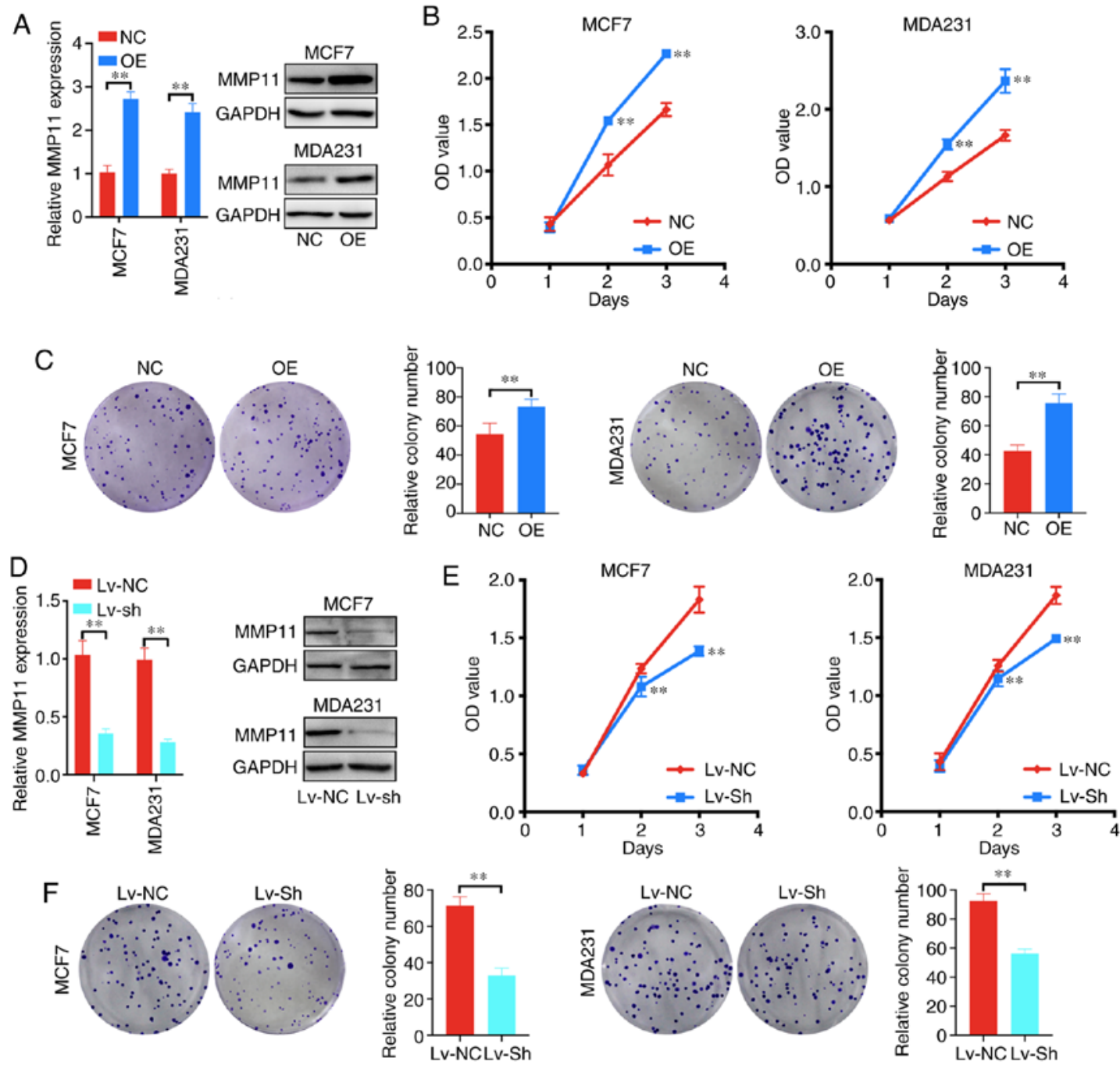

Figure 3. (A) RT-qPCR and western blotting revealed the expression of MMP11 in MCF7 and MDA231 cells transfected with the MMP11 OE plasmid and the corresponding NC plasmid. (B) The viability of MCF7 and MDA231 cells was assessed by MTT assay after cells were transfected with MMP11 OE plasmid and NC plasmid. (C) The proliferation capacity of MCF7 and MDA231 cells was assessed by colony formation assay after cells were transfected with MMP11 OE plasmid and NC plasmid. (D) RT-qPCR analysis revealed the expression of MMP11 in MCF7 and MDA231 cells transfected with a lentivirus containing MMP11 (LV-sh) or an empty lentiviral vector (LV-NC). (E) The viability of MCF7 and MDA231 cells was assessed by MTT assay after cells were transfected with a lentivirus containing LV-sh or LV-NC. (F) The proliferative capacity of MCF7 and MDA231 cells was assessed by colony formation assay after cells were transfected with a lentivirus containing LV-sh or LV-NC. All data are presented as the mean \pm SD of three independent experiments. ** $\mathrm{P}<0.01$. RT-qPCR, reverse transcription-quantitative PCR; MMP, matrix metalloproteinase; OE, overexpression; NC, negative control.

and this inhibition could not be increased by TGF- $\beta$ inducers (Fig. 4E and F), which revealed that MMP11 promoted the expression of Smad 2 and Smad3 independent on TGF- $\beta$.

MMP11 binds to Smad2 and inhibits its degradation. To verify the interaction of Smad2 and Smad3 with MMP11, first, changes in the mRNA expression of Smad2 and Smad3 were detected after MMP11 overexpression by RT-qPCR and it was revealed that there was no significant difference in the mRNA expression of $\mathrm{Smad} 2$ and $\mathrm{Smad} 3$ between the MMP11 OE group and the NC group (data not shown). It was further attempted to use anti-MMP11 antibodies to immunoprecipitate Smad2 or Smad3 from MCF7 cells, which exhibit endogenous expression of MMP11, Smad2 and Smad3. Notably, it was revealed that Smad2 was detected in the MMP11 immune complex by western blot analysis (Fig. 5A). Therefore, it was speculated that MMP11 could affect the protein degradation of Smad2. To further verify our conjecture, a CHX chase experiment was performed. The results revealed that transfection of MCF7 cells with MMP11 Lv-sh led to a marked reduction in the half-life of Smad2 compared with that of Lv-NC transfection (Fig. 5B), indicating that the decrease in MMP11 expression promoted the degradation of Smad2. Furthermore, MCF7 cells were transfected with MMP11 Lv-sh and then treated with the proteasome inhibitor MG132 or autophagy inhibitor chloroquine. The present results revealed that MG132 effectively abrogated the effect of MMP11 Lv-sh on Smad2 protein degradation, whereas chloroquine had no such effect (Fig. 5C and D), which confirmed that MMP11 inhibited Smad2 degradation through the ubiquitin proteasome pathway. Finally, the MMP11 OE plasmid and Smad2-siRNA were co-transfected into MCF7 cells, and the MMP11 overexpression-mediated induction of cell proliferation was significantly inhibited by si-Smad2 (Fig. 5E), which verified that Smad2 was a critical downstream factor that mediated the ability of MMP11 to promote BC cell proliferation. 
A

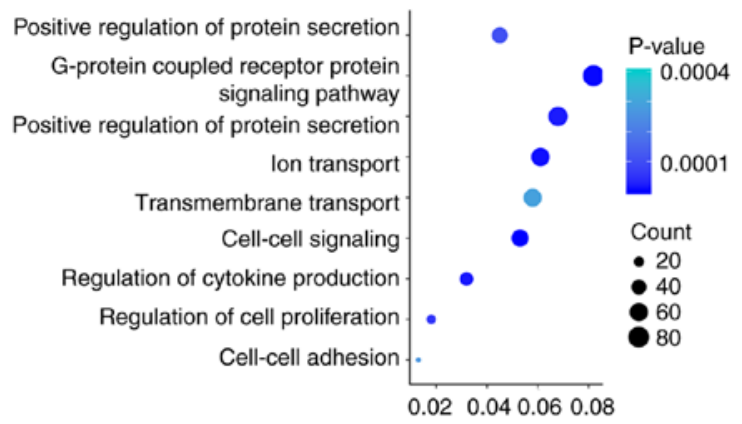

0.020 .040 .060 .08

B

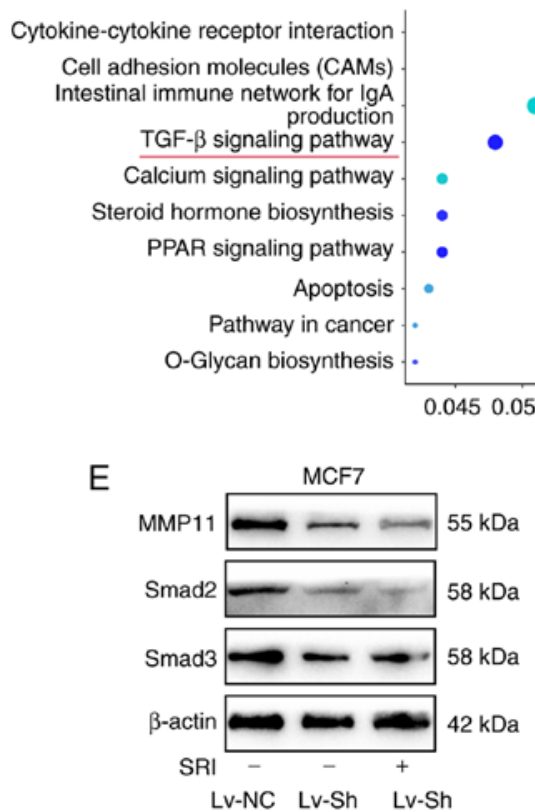

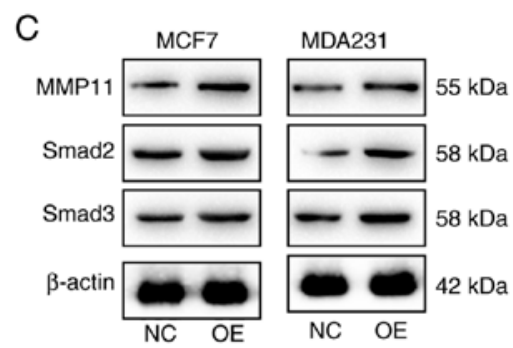

D

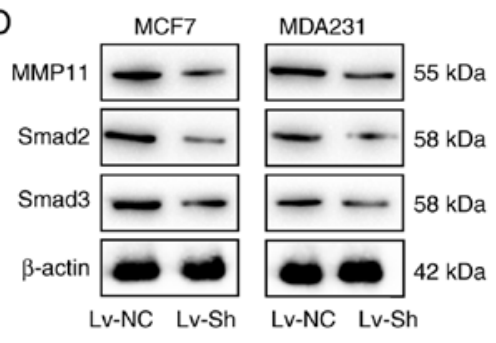

Figure 4. (A) BP analysis of MMP11-related molecules. (B) KEGG analysis of MMP11-related molecules. (C) Smad2 and Smad3 in the TGF- $\beta$ signalling pathway were detected by western blot assay after cells were transfected with MMP11 OE plasmid and NC plasmid. (D) Smad 2 and Smad3 in the TGF- $\beta$ signalling pathway was detected by western blot assay after cells were transfected with a lentivirus containing LV-sh or LV-NC. (E) MCF7 and (F) MDA231 were transfected with LV-sh or LV-NC, and processed by SRI as indicated, respectively. BP, biological process; MMP, matrix metalloproteinase; KEGG, Kyoto Encyclopedia of Genes and Genomes; OE, overexpression; NC, negative control; SRI, SRI-011381.

Decreased MMP11 inhibits BC growth in vivo. To further clarify the biological role of MMP11 in vivo, MCF7 cells stably transfected with Lv-NC or Lv-sh of MMP11 were implanted as xenografts in BALB/c nude mice (Fig. 6A). Compared with those in the Lv-NC group, the tumour weight and growth of the Lv-sh group were significantly inhibited (Fig. 6B and C). According to IHC, RT-qPCR and western blot assay results, the expression of MMP11 and Smad2 in implanted tumours of the Lv-sh group was significantly lower than that in the Lv-NC group (Fig. 6D-F). These results further indicated that MMP11 played an essential role in promoting BC through Smad2.

\section{Discussion}

$\mathrm{BC}$ treatment has come a long way in recent decades. However, $\mathrm{BC}$ is a challenging disease, and the OS rate has not significantly improved (18). If reliable potential molecular markers are available at the time of initial diagnosis, the prognosis and treatment outcomes of $\mathrm{BC}$ patients may be considerably improved. Therefore, there is a need to expand the current understanding of the molecular mechanisms of $\mathrm{BC}$ progression and to identify new biomarkers. There is ample evidence that MMP11 is a regulator of gene expression and complex pathways in cancer (19-21). In the present study, the relationship between MMP11 with clinicopathological parameters and total survival of BC was analysed. In addition, GO and KEGG pathway enrichment analyses were performed to assess gene function. Specifically, it was determined that high expression of MMP11 in BC was significantly associated with tumour stages and a poor prognosis. In addition, using bioinformatics analysis, it was demonstrated that MMP11 was associated with multiple signalling pathways related to tumour malignancy.

The present study first explored the biological roles of MMP11 in BC. A previous study have revealed that MMP11 is significantly associated with the regulation of cell proliferation in BC (22). The present study revealed that MMP11 was overexpressed at the mRNA and protein levels in BC tissues compared with PC tissues and that the expression levels of MMP11 in four PC cell lines was significantly higher than those in a normal human cell line. In addition, MMP11 overexpression was also revealed to significantly enhance the proliferative ability of MCF7 and MDA231 cells. To further demonstrate the roles of MMP11 in vivo, xenograft tumour formation experiments were 


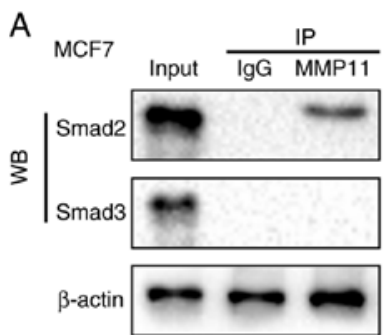

B
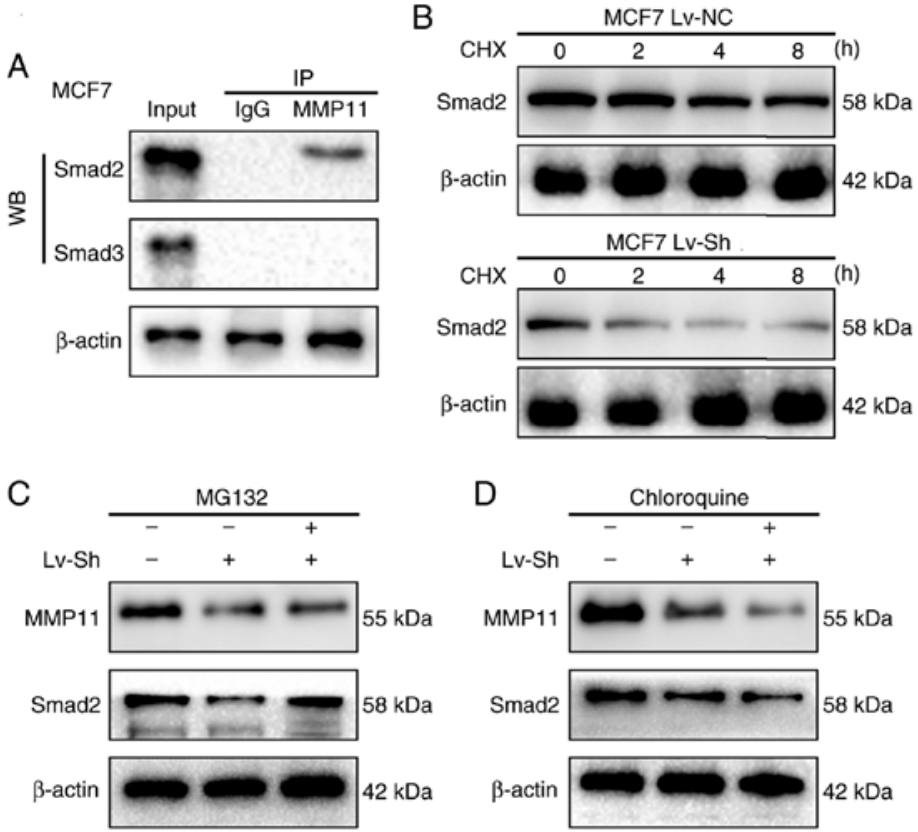

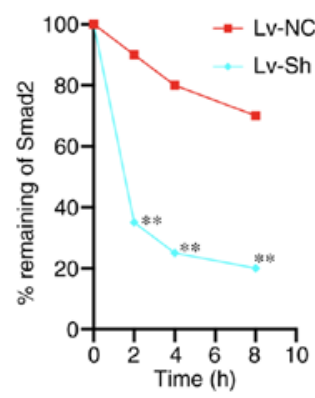

$\mathrm{E} \rightarrow \mathrm{NC}+$ si-NC
$\rightarrow \mathrm{NC}+\mathrm{Si}-\mathrm{Smad}$

$=\mathrm{OE}+\mathrm{Si}-\mathrm{NC}$

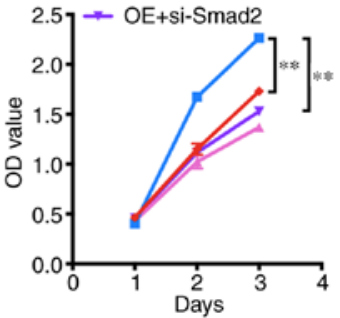

Figure 5. (A) Endogenous interaction between MMP11 and Smad2 was detected by western blot assay. (B) The Smad2 protein levels in MCF7 cells transfected with a lentivirus containing LV-sh or LV-NC were detected by western blotting assay at different time-points after exposure to the protein synthesis inhibitor CHX (10 mg/ml), as indicated. (C) Western blot assay of MCF7 cells transfected with a lentivirus containing LV-sh or LV-NC and treated with DMSO or $10 \mathrm{mM}$ MG132. (D) Western blot assay of MCF7 cells transfected with a lentivirus containing LV-sh or LV-NC and treated with DMSO or $10 \mathrm{mM}$ chloroquine. (E) The viability of MCF7 cells co-transfected with MMP11 OE plasmid and si-Smad2, as indicated, was assessed by MTT assay. All data are presented as the mean $\pm \mathrm{SD}$ of three independent experiments. ${ }^{* *} \mathrm{P}<0.01$. MMP, matrix metalloproteinase; $\mathrm{CHX}$, cycloheximide; $\mathrm{NC}$, negative control; OE, overexpression; si-Smad2, Smad2 siRNA.

A

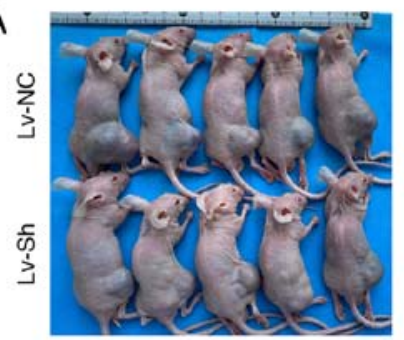

D

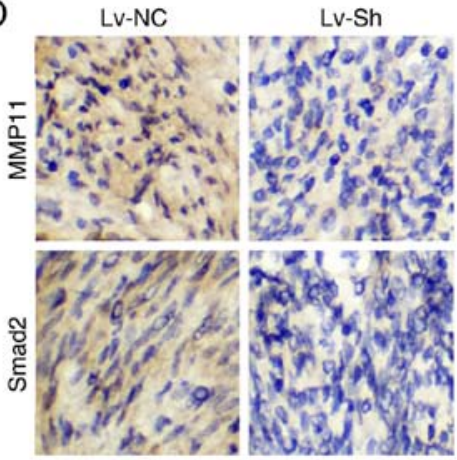

B

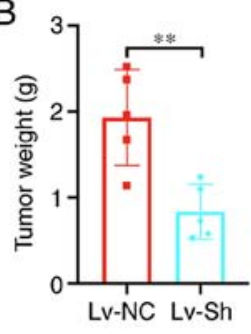

$\mathrm{E}$

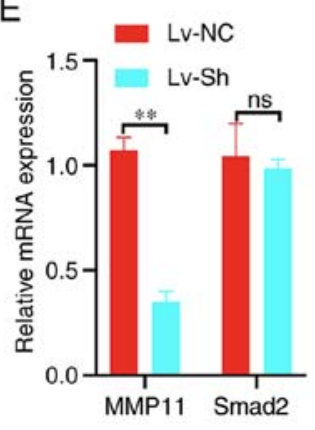

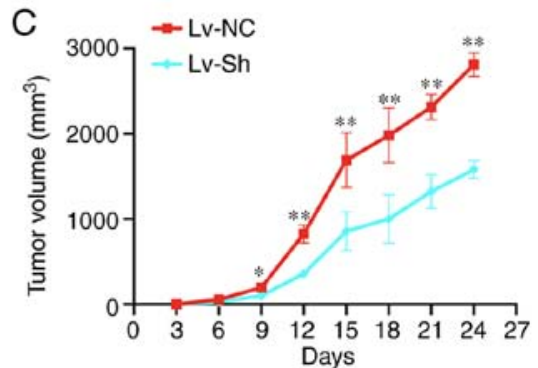

$\mathrm{F}$

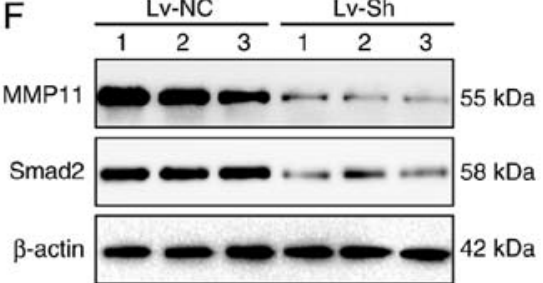

Figure 6. (A) Representative images of the nude mice and corresponding xenografts in the LV-sh and LV-NC groups 24 days after inoculation. (B) The tumour weight and (C) tumour volume in the LV-sh and LV-NC groups. The expression of MMP11 and Smad2 in implanted tumours was compared by (D) IHC assay, (E) RT-qPCR assay and $(\mathrm{F})$ western blot assay between the LV-sh and LV-NC groups. All data are presented as the mean \pm SD of three independent experiments. ${ }^{*} \mathrm{P}<0.05$ and $^{* *} \mathrm{P}<0.01$. NC, negative control; MMP, matrix metalloproteinase; IHC, immunohistochemistry; RT-qPCR, reverse transcription-quantitative PCR

conducted. The experimental results were consistent with those of the in vitro assays, that is, MMP11 knockdown significantly inhibited BC growth in vivo. These results further demonstrated the cancer-promoting role of MMP11 in BC.
To further explore the underlying mechanism, bioinformatics analysis was conducted and it was revealed that cell adhesion molecules played an important role in the process of MMP11 promoting $\mathrm{BC}$, however a previous study reported that they had 
a stronger relationship with tumour metastasis (23). Our initial purpose was to study tumour proliferation, thus, we first focused on the TGF- $\beta$ signalling pathway. TGF- $\beta$ superfamily signalling plays a critical role in the regulation of cell growth, differentiation, and development in a wide range of biological systems. In general, TGF- $\beta$ /activin signalling is initiated by ligand-induced oligomerization of serine/threonine receptor kinases and phosphorylation of the cytoplasmic signalling molecules Smad2 and Smad3. Moreover, in certain contexts, TGF- $\beta$ signalling could also affect Smad-independent pathways, including the MEK/Erk, SAPK/JNK, and p38 MAPK pathways (24-26). In the present study, mechanistically, it was revealed that MMP11 could significantly regulate the protein expression levels of Smad2 and Smad3. We first tried to explain its mechanism at the transcriptional level, however it was revealed that the mRNA levels of Smad2 and Smad3 were not significantly affected by changes in MMP11 expression. Based on the literature (5), it was also revealed that MMP11 does not function as a transcription factor. Therefore, it was hypothesized that MMP11 mainly affects the degradation of Smad2 and Smad3 protein. CHX and MG132 inhibition assays revealed that MMP11 could stabilize the Smad2 protein structure by inhibiting its ubiquitination-mediated degradation. Unfortunately, the direct connection between MMP11 and Smad3 has not been explored clearly, and this is the goal of our next study. In addition, notably, our analysis revealed that the aberrant expression of MMP11 was also related to cell adhesion molecules and apoptosis, which indicated that MMP11 played an important role in the development of BC.

In conclusion, the present results revealed that MMP-11 itself promoted $\mathrm{BC}$ cell proliferation, thereby promoting $\mathrm{BC}$ development. Mechanistically, the cancer-promoting roles of MMP11 in BC are related to the increased stability of the Smad 2 protein and the activation of the TGF- $\beta$ signalling pathway. Conversely, MMP11 inhibition in BC cells greatly impaired their ability to proliferate and grow. Moreover, it was concluded that the knockdown of MMP11 could reverse its pro-tumorigenic effects in vivo. Therefore, the MMP11 gene is proposed as a target for BC therapy.

\section{Acknowledgements}

Not applicable.

\section{Funding}

No funding was received.

\section{Availability of data and materials}

The datasets used and analysed during the current study were made available by the TCGA Genome Data Analysis Center of the Broad Institute in 2016. All other datasets used during the present study are available from the corresponding author upon reasonable request.

\section{Authors' contributions}

GW conceived and designed the study. YZ participated in the design of the study and performed bioinformatics analysis. $\mathrm{XL}, \mathrm{PZ}$, and GP provided their advice during the process of the research. YZ and XL performed the data analysis and wrote the manuscript. YZ, PZ, and GP performed the cell line validation assay. All authors reviewed and edited the manuscript and read and approved the final manuscript.

\section{Ethics approval and consent to participate}

All clinical samples were collected with written informed consent from the patients, and this protocol was approved by the Ethics Committee of the Hubei Cancer Hospital (Wuhan, China). The mouse experiments and the handling of animals were performed according to the Institutional and Animal Care and Use Committee of Hubei Cancer Hospital and the NIH Guide for the Care and Use of Laboratory Animals.

\section{Patient consent for publication}

Not applicable.

\section{Patient competing interests}

The authors declare that they have no competing interests.

\section{References}

1. Ferlay J, Colombet M, Soerjomataram I, Dyba T, Randi G, Bettio M, Gavin A, Visser O and Bray F: Cancer incidence and mortality patterns in Europe: Estimates for 40 countries and 25 major cancers in 2018. Eur J Cancer 103: 356-387, 2018.

2. Torre LA, Siegel RL, Ward EM and Jemal A: Global cancer incidence and mortality rates and trends-an update. Cancer Epidemiol Biomarkers Prev 25: 16-27, 2016.

3. Liu N, Johnson KJ and Ma CX: Male breast cancer: An updated surveillance, epidemiology, and end results data analysis. Clin Breast Cancer 18: e997-e1002, 2018.

4. Pittayapruek P, Meephansan J, Prapapan O, Komine M and Ohtsuki M: Role of matrix metalloproteinases in photoaging and photocarcinogenesis. Int J Mol Sci 17: 868, 2016.

5. Motrescu ER and Rio MC: Cancer cells, adipocytes and matrix metalloproteinase 11: A vicious tumor progression cycle. Biol Chem 389: 1037-1041, 2008.

6. Scheau C, Badarau IA, Costache R, Caruntu C, Mihai GL, Didilescu AC, Constantin C and Neagu M: The role of matrix metalloproteinases in the epithelial-mesenchymal transition of hepatocellular carcinoma. Anal Cell Pathol (Amst) 2019: 9423907, 2019

7. Zhang X, Huang S, Guo J, Zhou L, You L, Zhang T and Zhao Y: Insights into the distinct roles of MMP-11 in tumor biology and future therapeutics (Review). Int J Oncol 48: 1783-1793, 2016.

8. Han J, Choi YL, Kim H, Choi JY, Lee SK, Lee JE, Choi JS, Park S, Choi JS, Kim YD, et al: MMP11 and CD2 as novel prognostic factors in hormone receptor-negative, HER2-positive breast cancer. Breast Cancer Res Treat 164: 41-56, 2017.

9. Eiro N, Cid S, Fernández B, Fraile M, Cernea A, Sánchez R, Andicoechea A, DeAndrés Galiana EJ, González LO, Fernández-Muñiz Z, et al: MMP11 expression in intratumoral inflammatory cells in breast cancer. Histopathology 75: 916-930, 2019.

10. Roscilli G, Cappelletti M, De Vitis C, Ciliberto G, Napoli A, Ruco L, Mancini R and Aurisicchio L: Circulating MMP11 and specific antibody immune response in breast and prostate cancer patients. J Transl Med 12: 54, 2014.

11. Wang MW, Liu J, Liu Q, Xu QH, Li TF, Jin S and Xia TS: LncRNA SNHG7 promotes the proliferation and inhibits apoptosis of gastric cancer cells by repressing the P15 and P16 expression. Eur Rev Med Pharmacol Sci 21: 4613-4622, 2017.

12. Livak KJ and Schmittgen TD: Analysis of relative gene expression data using real-time quantitative PCR and the 2(-Delta Delta C(T)) method. Methods 25: 402-408, 2001. 
13. Goldman MJ, Craft B, Hastie M, Repečka K, McDade F, Kamath A, Banerjee A, Luo Y, Rogers D, Brooks AN, et al: Visualizing and interpreting cancer genomics data via the Xena platform. Nat Biotechnol 38: 675-678, 2020.

14. Györffy B, Lanczky A, Eklund AC, Denkert C, Budczies J, Li Q and Szallasi Z: An online survival analysis tool to rapidly assess the effect of 22,277 genes on breast cancer prognosis using microarray data of 1809 patients. Breast Cancer Res Treat 123: 725-731, 2010.

15. Huang da W, Sherman BT and Lempicki RA: Systematic and integrative analysis of large gene lists using DAVID bioinformatics resources. Nat Protoc 4: 44-57, 2009.

16. Huang da W, Sherman BT and Lempicki RA: Bioinformatics enrichment tools: paths toward the comprehensive functional analysis of large gene lists. Nucleic Acids Res 37: 1-13, 2009.

17. Chen L, Yang T, Lu DW, Zhao H, Feng YL, Chen H, Chen DQ, Vaziri ND and Zhao YY: Central role of dysregulation of TGF- $\beta /$ Smad in CKD progression and potential targets of its treatment. Biomed Pharmacother 101: 670-681, 2018.

18. Tao Z, Shi A, Lu C, Song T, Zhang Z and Zhao J: Breast cancer: Epidemiology and etiology. Cell Biochem Biophys 72: 333-338, 2015.

19. Li Z, Ding S, Zhong Q, Li G, Zhang Y and Huang XC: Significance of MMP11 and P14(ARF) expressions in clinical outcomes of patients with laryngeal cancer. Int J Clin Exp Med 8: 15581-15590, 2015.

20. Kou YB,Zhang SY,Zhao BL, Ding R,Liu H and Li S: Knockdown of MMP11 inhibits proliferation and invasion of gastric cancer cells. Int J Immunopathol Pharmacol 26: 361-370, 2013.
21. Han HB, Gu J, Zuo HJ, Chen ZG, Zhao W, Li M, Ji DB, Lu YY and Zhang ZQ: Let-7c functions as a metastasis suppressor by targeting MMP11 and PBX3 in colorectal cancer. J Pathol 226: 544-555, 2012.

22. González de Vega R, Clases D, Fernández-Sánchez ML, Eiró N, González LO, Vizoso FJ, Doble PA and Sanz-Medel A: MMP-11 as a biomarker for metastatic breast cancer by immunohistochemical-assisted imaging mass spectrometry. Anal Bioanal Chem 411: 639-646, 2019.

23. Janiszewska M, Primi MC and Izard T: Cell adhesion in cancer: Beyond the migration of single cells. J Biol Chem 295: 2495-2505, 2020.

24. Horbelt D, Denkis A and Knaus P: A portrait of transforming growth factor $\beta$ superfamily signalling: Background matters. Int J Biochem Cell Biol 44: 469-474, 2012.

25. Ikushima $\mathrm{H}$ and Miyazono K: TGFbeta signalling: A complex web in cancer progression. Nat Rev Cancer 10: 415-424, 2010.

26. Velapasamy S, Dawson CW, Young LS, Paterson IC and Yap LF: The dynamic roles of TGF- $\beta$ signalling in EBV-associated cancers. Cancers (Basel) 10: 247, 2018.

This work is licensed under a Creative Commons Attribution-NonCommercial-NoDerivatives 4.0 International (CC BY-NC-ND 4.0) License. 